\title{
WhatsApp in $m$ Health: design and evaluation of an $m$ Health tool to share dynamic images in hemodynamics
}

\author{
Daniele Giansanti ${ }^{1}$, Luca Cosentino ${ }^{2}$ \\ ${ }^{1}$ Centro TISP, Istituto Superiore di Sanità, Roma, Italia; ${ }^{2}$ Università Sapienza, Roma, Italia \\ Contributions: (I) Conception and design: All authors; (II) Administrative support: None; (III) Provision of study materials or patients: All authors; \\ (IV) Collection and assembly of data: All authors; (V) Data analysis and interpretation: D Giansanti; (VI) Manuscript writing: All authors; (VII) Final \\ approval of manuscript: All authors. \\ Correspondence to: Daniele Giansanti. Centro TISP, Istituto Superiore di Sanità, Roma, Italia. Email: daniele.giansanti@iss.it; Luca Cosentino. \\ Università Sapienza, Roma, Italia. Email: gianslele@gmail.com.
}

\begin{abstract}
Background: WhatsApp, like many other messaging Apps, enables messages, documents, and multimedia elements to be exchanged through a chat-based tool. Among the many potentialities of WhatsApp are: (I) the possibility to create groups (WhatsApp Groups) and (II) the possibility to install the app on a personal computer using the WhatsApp Web application.

Methods: The two functionalities (I, II) were used to set-up an $m$ Health environment for teleconsultation in telecardiology which will support the exchange of the dynamic images in hemodynamics. A proper client/ server architecture was proposed to supply the mHealth environment. The files related to the dynamic study were preliminarily converted [using picture archiving and communication system (PACS) functions] into an audio-video interleave (AVI) format, which is compatible with mobile technology.

Results: The mHealth environment was configured. Five dynamic images were extracted from the PACS in the format AVI. The images were exchanged using WhatsApp Web on the server side and WhatsApp on the client side (smartphones). An acceptance analysis of five observers showed high satisfaction with the methodology.

Conclusions: From a global perspective, our contribution aligns with those of scholars in the field concerning the use of WhatsApp in medical imaging. Furthermore, this study suggests that research in the telecardiology field can be strengthened through the use of dynamic images and WhatsApp.
\end{abstract}

Keywords: mHealth; heart; hemodinamics; WhatsApp; telemedicine

Received: 25 September 2019; Accepted: 11 June 2020; Published: 20 January 2021.

doi: $10.21037 /$ mhealth-19-187

View this article at: http://dx.doi.org/10.21037/mhealth-19-187

\section{Introduction}

\section{Tele-imaging today}

In the first decade of the $21^{\text {st }}$ century, contributions to the field of telemedicine were strongly linked to the types and architecture of the wired networks that supply information electronically $(1,2)$. Teleimaging is a tool that allows for histological and cytological and organ diagnostics remotely. In the high-tech $21^{\text {st }}$ century, there has been a technology boom which has allowed for the widespread use of such technology $(3,4)$. The field of organ diagnostics has achieved the highest degree of standardization of any of the remote diagnostic techniques. This high degree of standardization allows for the widespread use of remote telemedicine and e-health services in the field $(4,5)$. Today, these services are based on wired solutions and run with rigorous attention to quality standards and regulations (5). This applies to both static and dynamic medical images DMI)s, which must be archived using the standard "Digital Imaging and Communications in Medicine" (DICOM) criteria, which are: (I) the devices (CT, NMR, etc.) must be DICOM compliant and (II) the digital archives, the picture archiving 
and communication system (PACS), must be certified as medical devices, according to the regulations $(4,5)$.

\section{Moving to mobile-bealth}

However, since the end of 2010, smartphone and tabletbased mobile technology $(6,7)$ has grown in popularity. In general, compared to earlier technologies, smartphones and tablets have features with enormous technological potential that make them palatable for application in a telemedical setting; these include:

* Increased memory, higher computing capacity, much more advanced data connection capacity due to the presence of dedicated operating systems.

- Excellent production potential and management of multimedia content, such as taking high-resolution photos and producing video films.

* Easy-to-install applications,

* Most phones are equipped with a high-resolution touch screen.

* Virtual keyboards, which can be used to use/operate various device functions (from the address book to the notepad), the Internet, and installed applications, including social networks.

These potentialities, combined with the increasing use of the mobile networks and miniaturization processes, have facilitated the diffusion of mobile health (mHealth). Recent pilot studies have demonstrated the potentialities of WhatsApp for telemedicine imaging, as reported in the review (8). Williams and Kovarik, for instance, discussed the usefulness of WhatsApp in medical imaging $(8,9)$ in teledermatology. Other recent studies have also highlighted the opportunities WhatsApp presents as an adjunctive tool in mHealth (10-11), or for specific applications, such as with oral medicine (12), orthopedics (13), and tele-education (14). The review (8) also outlined the potentialities of WhatsApp in digital pathology (DP) (which comprises two components: digital cytology and digital histology) evolved from eHealth (15) to mHealth (16-18) (which comprises two components: digital cytology and digital histology) and in digital radiology (DR) (19). The possibility of using WhatsApp in relation to dynamic imaging needs to be investigated in depth.

\section{Study aims}

To date, the use of WhatsApp in medical imaging has mainly been limited to the exchange of static images.
However, medical diagnosis is also based on the kinetics of the investigated imaging process.

The objective of this pilot study is to investigate the exchange of dynamic hemodynamics images in mHealth with a specific focus on cardiology, using WhatsApp and adequately designed client-server-architecture (CSA), and to consult stakeholders on the new potentialities of the technology.

\section{Methods}

\section{The components of the client-server-architecture}

The two functionalities of WhatsApp used for the design of the CSA were:

* The function used to create a workgroup to manage the exchange of the images between the participants. A group named Digital Radiology Network was configured.

* WhatsApp Web as the sever installation (i.e., the platform with the PACS) to send images from here to the remote teleconsulting sides. Figure 1 shows WhatsApp Web in the CSA.

The methodological flow used to set-up the CSA is reported in Figure 2.

Figure 2 A flow chart showing the flow of the digital information from the software SuiteEstensa PACS (Esaote, Italy) (the server) (where the file of the hemodynamic study is stored) to the remote smartphone (the client).

The flow of digital information can be divided into three phases:

* The first phase consists of the extraction of the run relevant to the hemodynamic study from the PACS, and its conversion into an audio-video interleave (AVI) file format. Figure 3 highlights the extraction of the file from the PACS managed by the SuiteEstensa software. A proper setting was used to guarantee the patient's privacy.

* The second phase consists of the insertion of the AVI file into the Google Drive cloud (Figure 2).

* The third phase consists of: (I) generating the link generation and sending it using WhatsApp Web at the server side and (II) receiving and opening the link using WhatsApp at the client side (Figure 2).

The study did not directly involve any human subjects. Only anonymous images of past processed trials were used. The ethical approval was not judged necessary. The consent forms were not therefore applicable. 


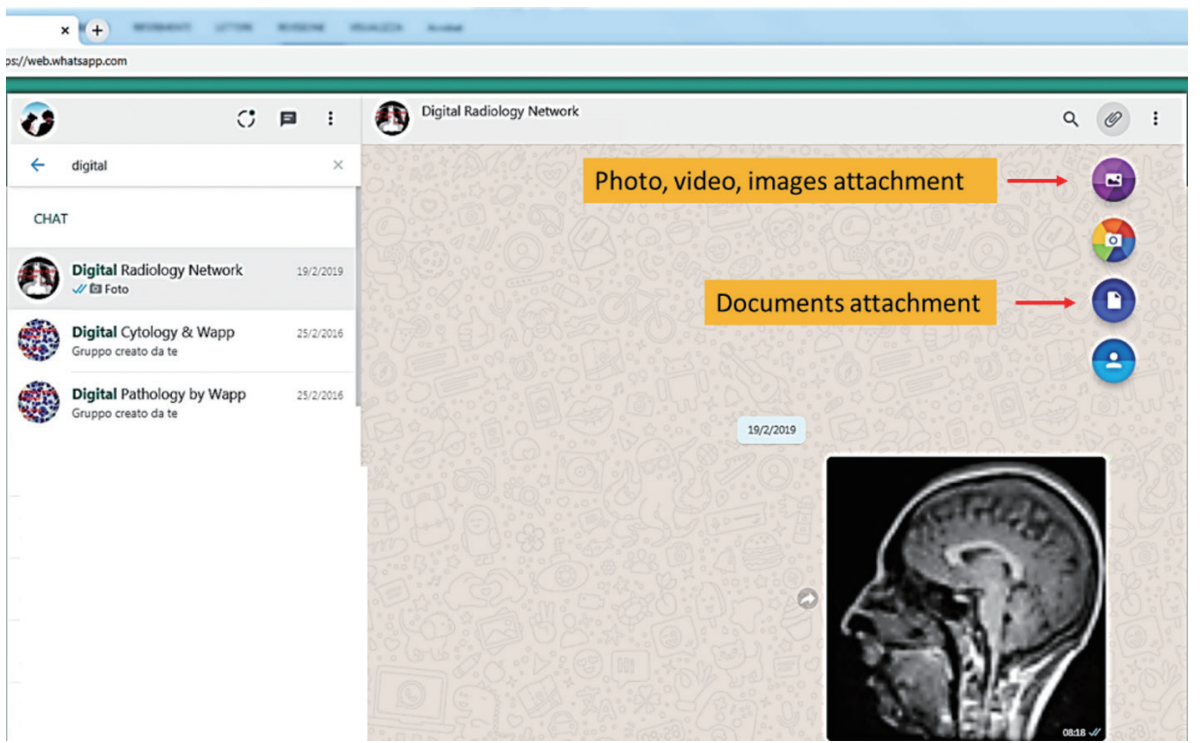

Figure 1 WhatsApp Web: a part of the CSA at the server side; it was also visible to the group participants. CSA, client-server-architecture.

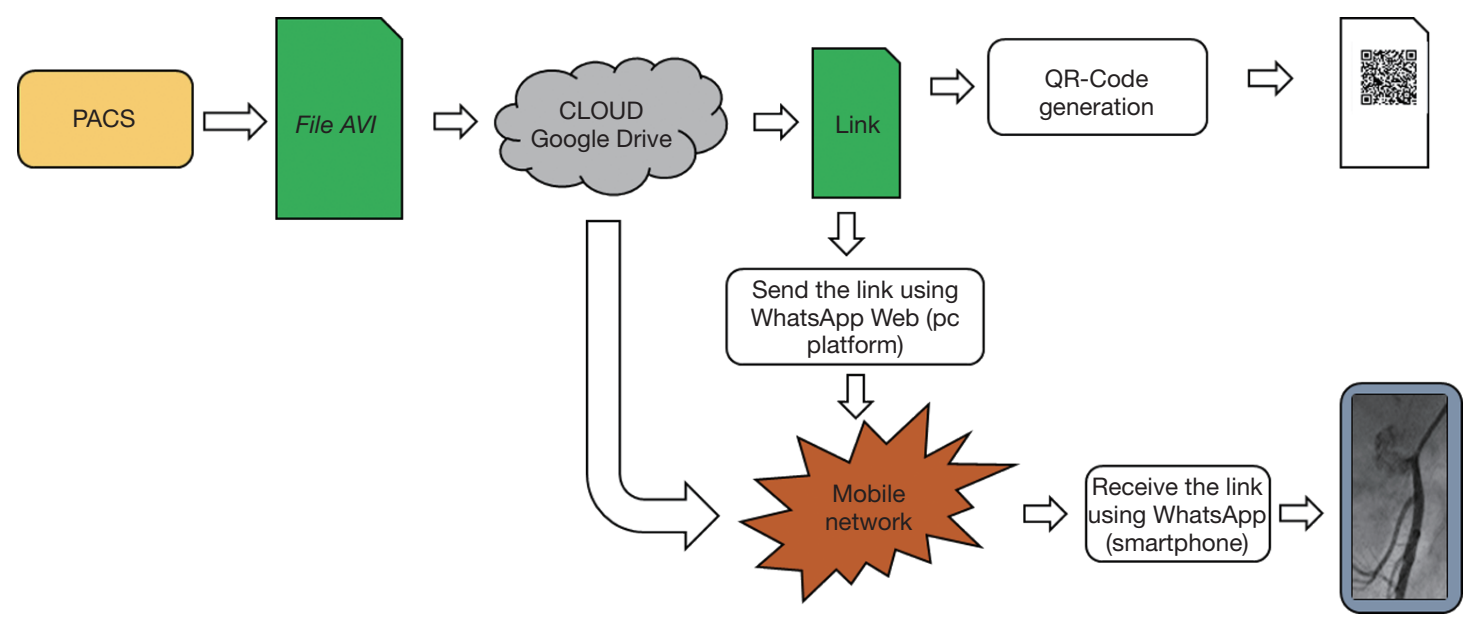

Figure 2 Flow chart showing the CSA flow. CSA, client-server-architecture.

\section{A Sample of a shared run}

Figures 4 and 5 show a sample of a shared run of a hemodynamic study. Figure 4 shows two screenshots of the run on the client side (smartphone) before and the "play button" was pressed. Figure 5 shows the link, and the Quick Response $(\mathrm{QR})$ code of the run shared via cloud. The dynamic image can be shared on WhatsApp by scanning the QR code below.

\section{Results}

The flow based on the CSA has been evaluated in a dedicated study conducted at the University Sapienza in Roma. The study involved three participants: one medical perfusionist in cardiology, one PACS system administrator, and one bioengineer. The first two participants selected the resources for the test: five dynamic images from five cardiologic studies (runs) in hemodynamics relevant to subjects with myocardial infarction. The system administrator managed the digital data according to the flow (Figure 2) from the PACS stage up to the link being sent via WhatsApp. Data were managed according to the security and privacy regulations. This study did not change/alter 

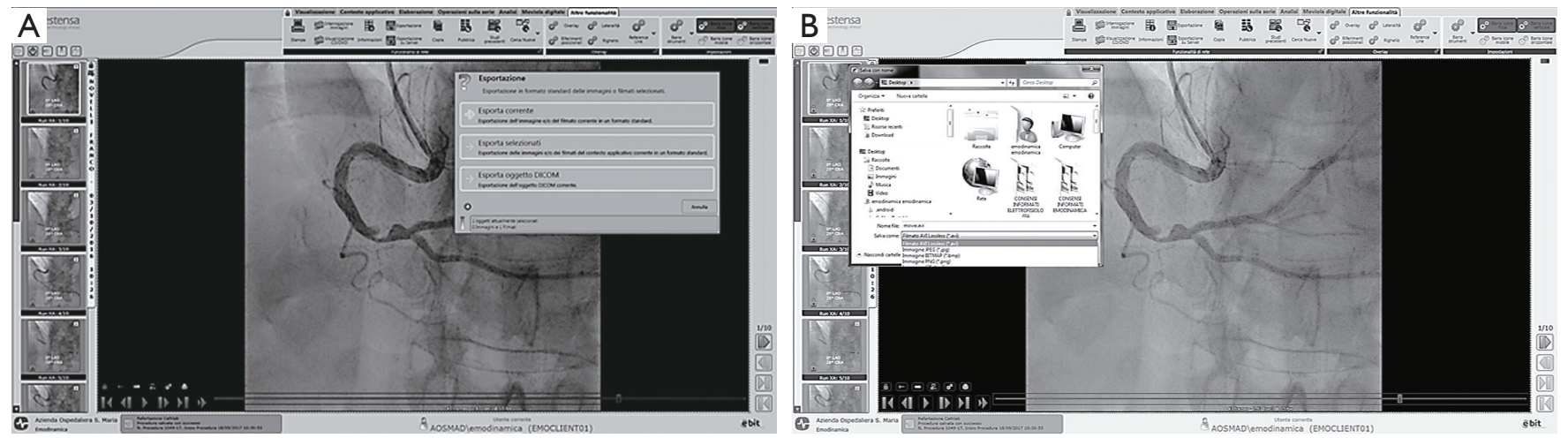

Figure 3 Extraction of the study from the SuiteEstensa (another part of the CSA at the server side): (A) The file was exported; (B) The selection of the imaging format for the export. CSA, client-server-architecture.
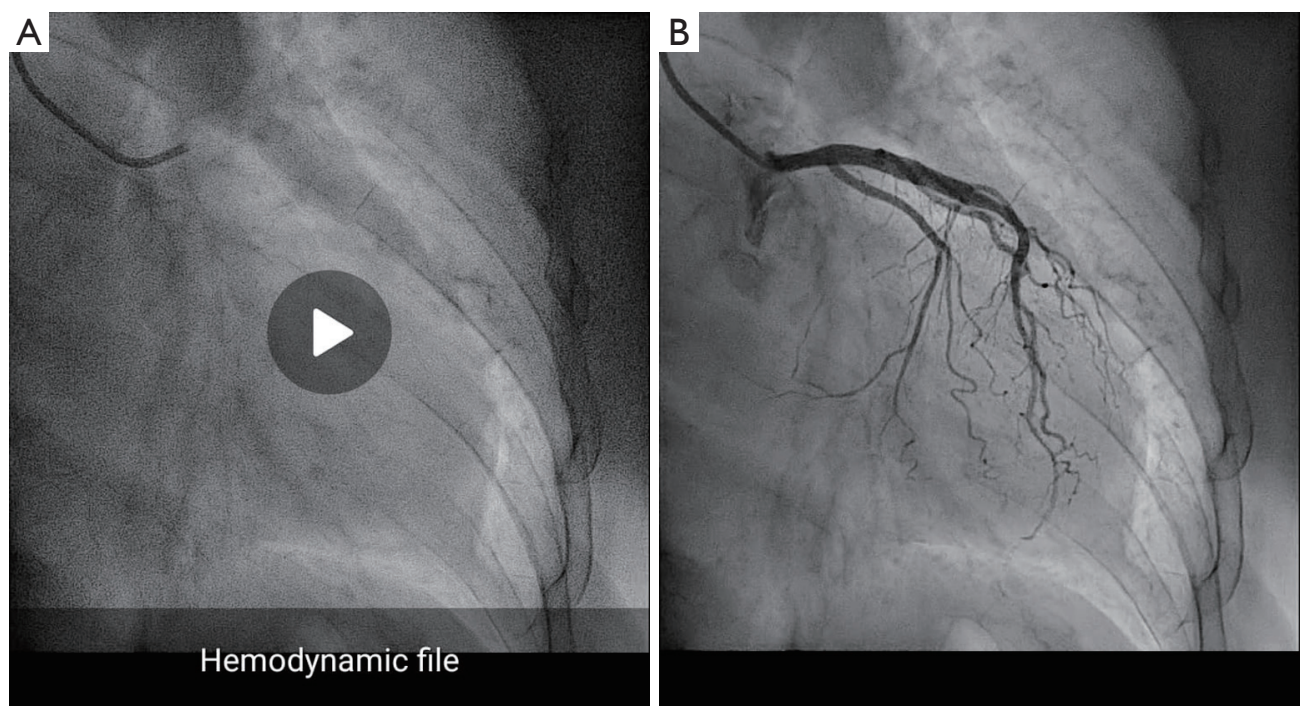

Figure 4 The sharing of the run: (A) the hemodynamic file and (B) the hemodynamic file opened after the the "play button" was pressed at the client side.

the hospital procedures; indeed, the runs have been stored previously during the routine activity (based on standardized procedures) according to the standard DICOM. The functions of the PACS were based on SuiteEstensa. Then, the extraction of the run with a conversion from DICOM into an AVI format was performed. The bioengineer and the system administrator designed the flow.

A survey was designed and conducted. It discussed five questions on the acceptance of the method based on the CSA. It was submitted to five experts/observers in the field of interventional radiology.

Each question includes a graded evaluation $(\min =1 ; \max$ =4). The following aspects were evaluated:

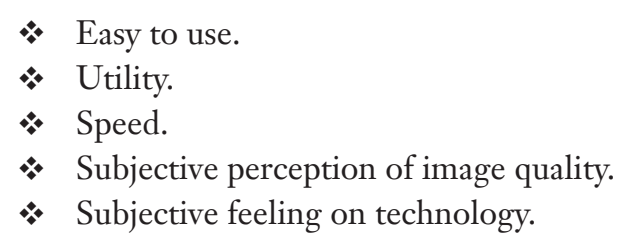

The test was conducted with the smartphone LG model K10 (LG Group, South Corea) with android OS and the basic functions (chosen because it represents a product of the low cost of about $€ 100$ ) in an area with at least a $3 \mathrm{G}$ wireless field with $70 \%$ power. Figure 6 shows encouraging results:

* The first three aspects were rated above 3.8 on average. 
* The two aspects related to feeling (I) subjective perception of the quality of the image and (II) subjective perception of technology in were rated 3 on average.

The experts were also asked which sector they think the method could be best applied.

All of the experts also identified "the future sectors where to deepen the investigation of the application of the methodology,"; identifying, for example, as a possible the echography.

\section{Discussion}

This work proposes a CSA for the exchange of DMIs using WhatsApp. The CSA was used in cardiology to exchange the DMIs in haemodynamics. Five images were exchanged. The method was successfully assessed by five experts/observers. From a global perspective, this work shows how instant messaging, which is mostly used in social networks, could contribute to mobile technology-based imaging teleconsulting. In particular, the study focused on hemodynamics and using WhatsApp for teleconsultation.

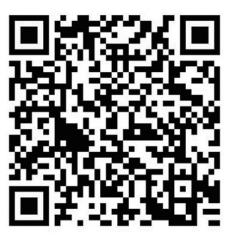

Figure 5 The Quick Response code of the sample.
It was also possible for DMIs to be exchanged. The preliminary results show that the methodology could prove to be an outstanding contribution in the field of telemedicine and e-Health delivered using the mHealth. The creation of a dedicated study on health technology assessment (HTA) should supply indications for stabilizing and spreading the methodology. At the moment, we are moving into two directions: first, we are planning a specific environment for the evaluation of the HTA of the methodology, beginning with a previous approach designed in other studies (15-17,20-22), and secondly, we are focusing on the field of hemodynamics and echography where, as suggested by this study there are important perspectives (8). While this study shows the usefulness of WhatsApp in this field, the output is not ready for direct use in routine diagnostics. Much work is needed to ensure the CSA is accepted by the scientific community and meets adequate regulations. However, this study offers important direction for pilot research studies directly related to the application of social media in medical imaging. The immediate usefulness of the study in clinical care is not focused on the direct diagnosis of patients; it is the sector of medical training (for example for the residents) on the implementation of remote teleimaging. This method could be useful for designing solutions for e-learning in this field for DP (15-17). This could contribute to free hospital resources for strategic activities related to both direct and interventional diagnosis, as in the case of the DP.

\section{Conclusions}

The study proposed a CSA flow to exchange the runs in

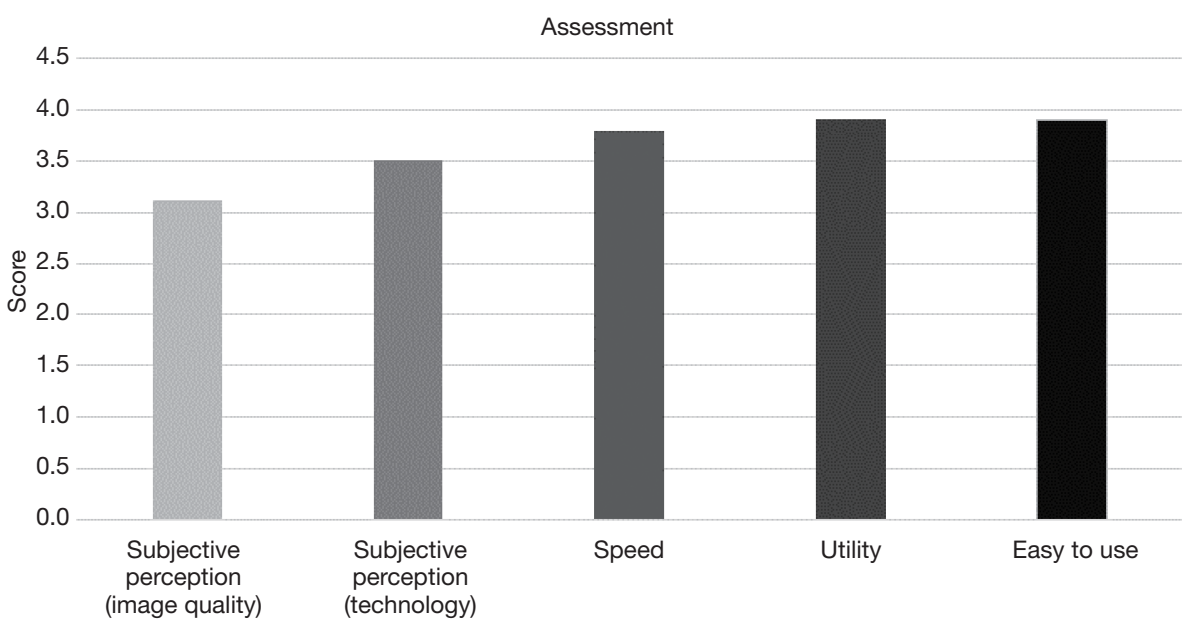

Figure 6 The experts' assessment of the method. 
hemodynamics using WhatsApp. Five dynamic images were extracted from PACS in an AVI format and were used to evaluate the procedure. The acceptance analysis conducted on five observers showed a very high satisfaction rate with the method. From a global perspective, this contribution aligns with those of scholars in the field in relation to the use of WhatsApp in medical imaging (8).

The general added value of this work is that the contribution it makes is consistent with other studies on the possibilities of using WhatsApp for the exchange of medical images. The study is in complete agreement with the proposals of previous studies $(8,18,19)$ on the usefulness, potentialities, and perspectives of WhatsApp.

(I) The first added value is of the defined flow of the CSA integrating eHealth and mHealth.

(II) The second added value is that the study on the acceptance of the CSA was conducted on five observers and featured five different dynamic images. This study, based on designed parameters, achieved a high, encouraging acceptance rate.

(III) The third added value is that the method could be applied in clinical care training, a field not focused on direct diagnosis. As in the case of the DP, this methodology could be useful in the design of solutions for e-learning in this field (15-17), which could free hospital resources for strategic activities related to direct and interventional diagnosis.

(IV) The fourth added value is that experts have forecasted the evolution of the use of WhatsApp in some fields of medical imaging.

\section{Acknowledgments}

Funding: None.

\section{Footnote}

Provenance and Peer Review: This article was commissioned by the Guest Editors (Sheikh Mohammed Shariful Islam and Ralph Maddison) for the series "Digital Health for Cardiovascular Disease" published in the mHealth. The article was sent for external peer review organized by the Guest Editors and the editorial office.

Conflicts of Interest: All authors have completed the ICMJE uniform disclosure form (available at http://dx.doi. org/10.21037/mhealth-19-187). The series "Digital Health for Cardiovascular Disease" was commissioned by the editorial office without any funding or sponsorship. Daniele Giansanti serves as an unpaid editorial board member of the mHealth from Apr 2019 to Apr 2021. The authors have no other conflicts of interest to declare.

Ethical Statement: The authors are accountable for all aspects of the work in ensuring that questions related to the accuracy or integrity of any part of the work are appropriately investigated and resolved. The study did not directly involve any human subjects. Only anonymous images of past processed trials were used. The ethical approval was not judged necessary. The consent forms were not therefore applicable.

Open Access Statement: This is an Open Access article distributed in accordance with the Creative Commons Attribution-NonCommercial-NoDerivs 4.0 International License (CC BY-NC-ND 4.0), which permits the noncommercial replication and distribution of the article with the strict proviso that no changes or edits are made and the original work is properly cited (including links to both the formal publication through the relevant DOI and the license). See: https://creativecommons.org/licenses/by-nc-nd/4.0/.

\section{References}

1. Giansanti D, Grigioni M, Giovagnoli MR. Virtual microscopy and digital cytology: fact or fantasy? Preface. Ann Ist Super Sanita 2010;46:113-4.

2. Giansanti D, Morelli S. Digital tele-echocardiography: a look inside. Ann Ist Super Sanita 2009;45:357-62.

3. Giansanti D. editors. Digital cytology: an experience with image-enhancement and tablet technologies. Roma: Istituto Superiore di Sanità; 2016. (Rapporti ISTISAN 16/46).

4. Giansanti D. editors. Diagnostic imaging and e-health: standardization, experiences and new opportunities. Roma: Istituto Superiore di Sanità; 2017. (Rapporti ISTISAN 17/10) (in italan).

5. Giansanti D. Teleradiology Today: The Quality Concept and the Italian Point of View. Telemed J E Health 2017;23:453-5.

6. Giansanti D, Grigioni M. editors. La salute in un palmo di mano: nuovi rischi da abuso di tecnologia. Roma: Istituto Superiore di Sanità; 2018. (Rapporti ISTISAN 18/21).

7. Giansanti D. editors. La salute in un palmo di mano: tra opportunità e problematiche. Roma: Istituto Superiore di Sanità; 2019. (Rapporti ISTISAN 19/15). 
8. Giansanti D. WhatsApp in mHealth: an overview on the potentialities and the opportunities in medical imaging. mHealth 2020;6:19.

9. Williams V, Kovarik C. WhatsApp: An Innovative Tool for Dermatology Care in Limited Resource Settings. Telemed J E Health 2018;24:464-8.

10. Mars M, Scott RE. Being spontaneous: The future of telehealth implementation? Telemed J E Health 2017;23:766-72.

11. Giordano V, Koch H, Godoy-Santos A, et al. WhatsApp Messenger as an Adjunctive Tool for Telemedicine: An Overview. Interact J Med Res 2017;6:e11.

12. Petruzzi M, De Benedittis $M$. WhatsApp: a telemedicine platform for facilitating remote oral medicine consultation and improving clinical examinations. Oral Surg Oral Med Oral Pathol Oral Radiol 2016;121:248-54.

13. Ellanti P, Moriarty A, Coughlan F, et al. The Use of WhatsApp Smartphone Messaging Improves Communication Efficiency within an Orthopaedic Surgery Team. Cureus 2017;9:e1040.

14. Coleman E, O'Connor E. The role of WhatsApp ${ }^{\circledR}$ in medical education; a scoping review and instructional design model. BMC Med Educ 2019;19:279.

15. Giansanti D, Castrichella L, Giovagnoli MR. The design of a health technology assessment system in telepathology. Telemed J E Health 2008;14:570-5.

16. Giansanti D, Pochini M, Giovagnoli MR. Integration

doi: 10.21037/mhealth-19-187

Cite this article as: Giansanti D, Cosentino L. WhatsApp in mHealth: design and evaluation of an mHealth tool to share dynamic images in hemodynamics. mHealth 2021;7:9. of tablet technologies in the e-laboratory of cytology: a health technology assessment. Telemed J E Health 2014;20:909-15.

17. Giansanti D, Pochini M, Giovagnoli MR. How tablet technology is going to change cooperative diagnosis in the cytology e-laboratory. Telemed J E Health 2013;19:991-3.

18. Giansanti D. WhatsApp in digital cytology teleconsulting: a preliminary assessment. In: Giansanti D. editors. Digital cytology: an experience with image-enhancement and tablet technologies. Roma: Istituto Superiore di Sanità; 2016. (Rapporti ISTISAN 16/46).

19. Giansanti D. Use of WhatsApp in digital radiology: preliminary considerations. In: Giansanti D. editors. Diagnostic imaging and e-health: standardization, experiences and new opportunities. Roma: Istituto Superiore di Sanità; 2017. (Rapporti ISTISAN 17/10) (in italan).

20. Giansanti D, Morelli S, Macellari V. A protocol for the assessment of diagnostic accuracy in teleechocardiography imaging. Telemed J E Health 2007;13:399-405.

21. Giansanti D, Morelli S, Macellari V. Telemedicine technology assessment part II: tools for a quality control system. Telemed J E Health 2007;13:130-40.

22. Giansanti D, Morelli S, Macellari V. Telemedicine technology assessment part I: setup and validation of a quality control system. Telemed J E Health 2007;13:118-29. 